\title{
Apparatus to measure high-temperature thermal conductivity and thermoelectric power of small specimens
}

\author{
T. Dasgupta and A. M. Umarji ${ }^{\text {a) }}$ \\ Materials Research Centre, Indian Institute of Science, Bangalore-560012, India
}

\begin{abstract}
A thermal conductivity apparatus based on the parallel heat-flow technique has been fabricated to measure the thermal conductivity of small specimens. The steady-state method is used to measure samples of $1 \times 1 \times 10 \mathrm{~mm}^{3}$ dimension in the temperature range of room temperature- $700 \mathrm{~K}$. The details of instrument fabrication, the method of calibration, and typical measurements on test samples are described. The apparatus can also be used to measure the Seebeck coefficient in the same temperature range. As an example we report the thermal properties of $\mathrm{CrSi}_{2}$, which is a potential candidate for high-temperature thermoelectric applications.
\end{abstract}

\section{INTRODUCTION}

In recent years there has been a considerable increase in the effort to identify new and efficient thermoelectric materials that would supercede the existing materials, especially at higher temperatures. For a material to be considered as a good thermoelectric, it must have a large figure of merit defined as $\mathbf{Z}=\mathbf{S}^{2} \boldsymbol{\sigma} / \mathbf{K}$, where $\mathbf{S}$ is the Seebeck coefficient, $\boldsymbol{\sigma}$ is the electrical conductivity, and $\mathbf{K}$ is the thermal conductivity. Amongst these three properties, $\mathbf{K}$ is most difficult to measure experimentally.

For relatively big samples there are well-established, steady-state and nonequilibrium techniques, ${ }^{1-3}$ but small samples pose difficulties associated with heat flow during the measurement. The most widely used commercial method is based on the laser flash technique. ${ }^{3}$ But the method has limitations for high $\mathbf{K}$ materials and at elevated temperatures. Recently Tritt et al. ${ }^{4}$ described a method known as the parallel conductance technique, which is suitable for small samples. They measured the $\mathbf{K}$ value for a few standard materials from $10 \mathrm{~K}$ to room temperature.

The problems associated with heat loss increases with temperature and measurement of $\mathbf{K}$ at high temperatures is very challenging. In this paper we describe an apparatus, which can measure the $\mathbf{K}$ value of small samples $(1 \mathrm{~mm}$ $\times 1 \mathrm{~mm} \times 10 \mathrm{~mm}$ ) based on the parallel conductance technique from room temperature to $700 \mathrm{~K}$. The same apparatus can also be used to measure the Seebeck coefficient of the material without disturbing the physical mounting.

The paper is divided into three sections. In Sec. II, the principle of measurement is explained, followed by a description of the apparatus in Sec. III. Section IV contains results obtained for standard samples.

\footnotetext{
${ }^{\text {a) }}$ Author to whom correspondence should be addressed; electronic mail: umarji@mrc.iisc.ernet.in
}

\section{PRINCIPLE OF MEASUREMENT}

\section{A. Thermal conductivity measurement}

Under steady-state conditions the $\mathbf{K}$ of a material is defined as

$$
\mathbf{K}=\left(Q_{S}^{\prime} / A\right) /(\Delta T / \Delta L),
$$

where $Q_{S}^{\prime}$ is the amount of heat passing per unit time through a cross-section area $A$, and causing a temperature difference, $\Delta T$, over a distance $\Delta L . Q_{S}^{\prime} / A$ is therefore the heat flux that is causing the temperature gradient, $\Delta T / \Delta L$. The measurement of thermal conductivity therefore involves the measurement of the heat flux and temperature difference. The difficulty of the measurement is always associated with heat-flux measurements because of the undefined amount of heat loss accompanying the heat transfer process.

A schematic representation of a heater block-sink assembly is depicted without and with the sample in Figs. 1(a) and 1(b), respectively. It is a steady-state method where temperature differences between the source and sink are measured as a function of time and the sample holder and sample act as two parallel heat conducting paths. First, the characteristics of the sample holder are measured, which determines the baseline or background thermal conduction and losses associated with the sample stage. The second step consists of attaching the sample and measuring the new characteristics of the assembly. By subtraction of the background thermal conduction, the thermal conductivity of the sample is calculated.

When heat is supplied to the heat source of Fig. 1(a), a part of it is utilized in raising the temperature of the source block while the remaining is dissipated to the surrounding through the cell assembly. At a constant input power, the temperature initially rises linearly as a function of time as the heat loss is negligible. Subsequently, as the temperature increases, the heat loss also increases and at equilibrium both the input power and the heat loss per unit time become equal. The temperature of the heater block at equilibrium reaches a constant value, which is taken as the equilibrium $\Delta T$. Thus 


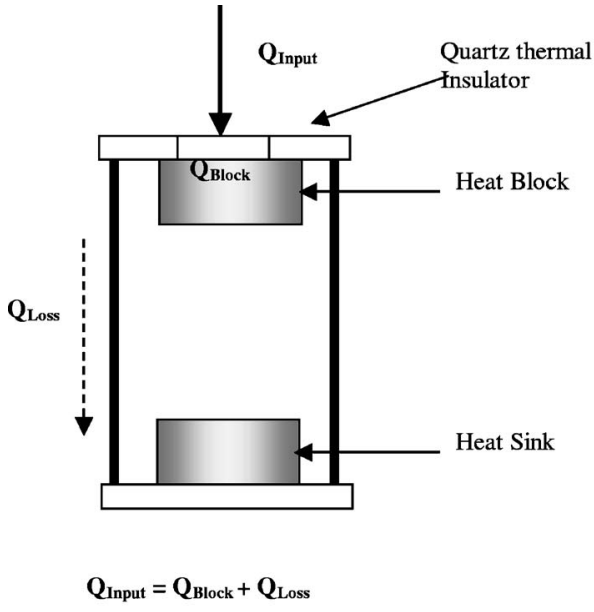

(a)

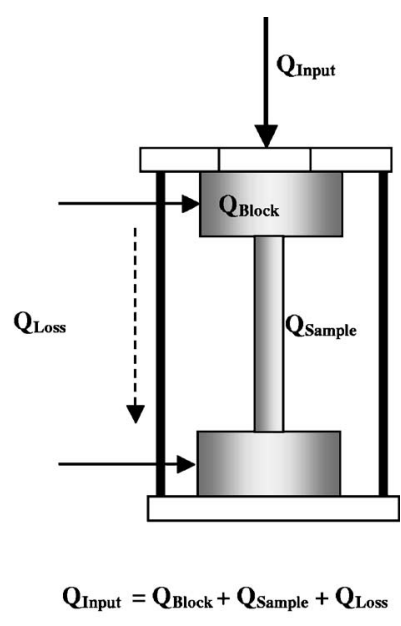

(b) the total heat input is a summation of the heat gained by the source and the heat dissipated to the surrounding. When a sample is placed between the source sink assembly [Fig. 1(b)], a part of the heat passes through the sample, which depends on the $\mathbf{K}$ of the sample. Thus the heat input is distributed among the heating source block, the heat transfer through the sample, and heat loss through the cell assembly. If the same heat input is maintained in both the cases and assuming that the heat dissipated in both is the same, the $\mathbf{K}$ of the sample can be found. This is known as the parallel conductance method.

Thus mathematically, it can be written as follows: Without any sample [as in Fig 1(a)],

$$
P_{E}=x \Delta T_{E}+H^{\prime}
$$

with the sample [as in Fig 1(b)],

$$
P_{S}=x \Delta T_{S}+H^{\prime}+Q_{S}^{\prime},
$$

where the subscripts $E$ stand for an empty run (i.e., without the sample), $S$ stand for a run with the sample. $P_{E}$ and $P_{S}$ is the power supplied, $\Delta T_{E}$ is the equilibrium temperature change in the heater block without the sample, and $\Delta T_{S}$ is the equilibrium temperature change with the sample. $H^{\prime}$ is the heat loss per unit time. $x$ is the heater constant that depends on the specific heat, mass, etc. of the heater block. $Q_{S}^{\prime}$ is the heat flux through the sample.

In Eq. (2) there are two unknowns, $x$ and $H^{\prime}$. The value of the heater constant $(x)$ has been obtained from the plot of $\Delta T_{E}$ vs $P$. This is explained in Sec. IV. The other unknown, $H^{\prime}$, can be assumed to be the same for a constant input power (i.e., $P_{E}=P_{S}$ ). If the same power is passed for both and assuming $H^{\prime}$ is the same, Eqs. (2) and (3) can be equated to the following:

$$
x \Delta T_{E}+H^{\prime}=x \Delta T_{S}+H^{\prime}+Q_{S}^{\prime} .
$$

Rearranging Eq. (4),

$$
Q_{S}^{\prime}=x\left(\Delta T_{E}-\Delta T_{S}\right) .
$$

Thus, once the heat flux through the sample $\left(Q_{S}^{\prime}\right)$ is known, the $\mathbf{K}$ of the material can be calculated using Eq. (1).

\section{APPARATUS DESCRIPTION}

A schematic diagram of the apparatus is shown in Fig. 2. The specimen (1) is placed inside a copper sample holder assembly. The sample holder consists of two copper $10 \mathrm{~mm}$ cylindrical blocks (2 and 3 ) into which type-K thermocouples (4) of 40 Imperial standard wire gauge (swg) are embedded. The thermocouples are in good thermal contact but electrically insulated from the blocks using silica sheaths. The crucial heat source copper block (2) is a $10-\mathrm{mm}$ diameter solid cylinder (weighing $10 \mathrm{~g}$ ) on which $20 \Omega$ of kanthal (32 swg) heater is wound. The heater is electrically insulated from the copper block using high-temperature cement. The bottom heat-sink block (3) is welded to the copper

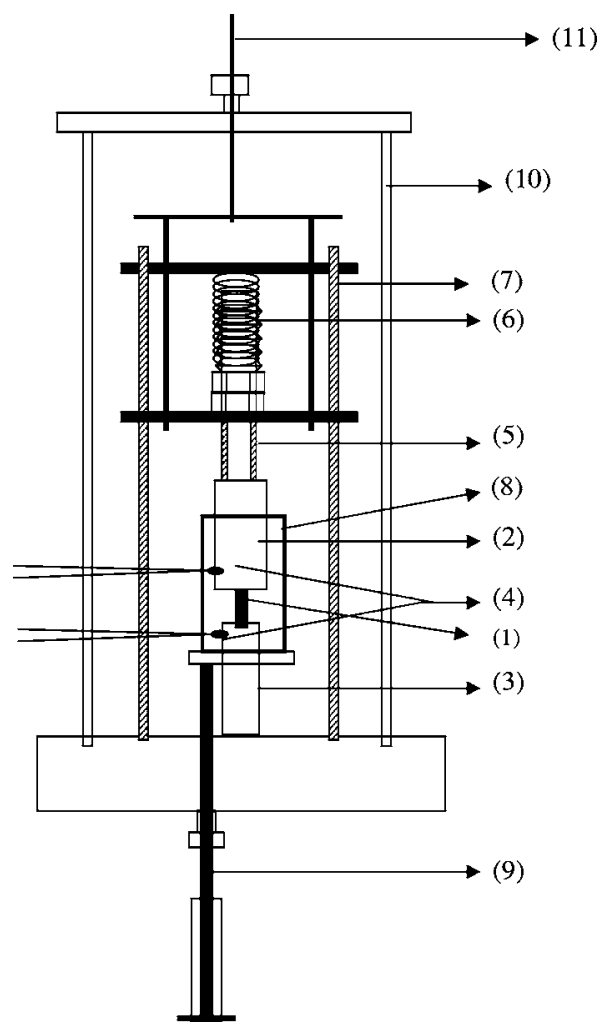

FIG. 2. Schematic diagram of the thermal conductivity setup (see text for explanations). 


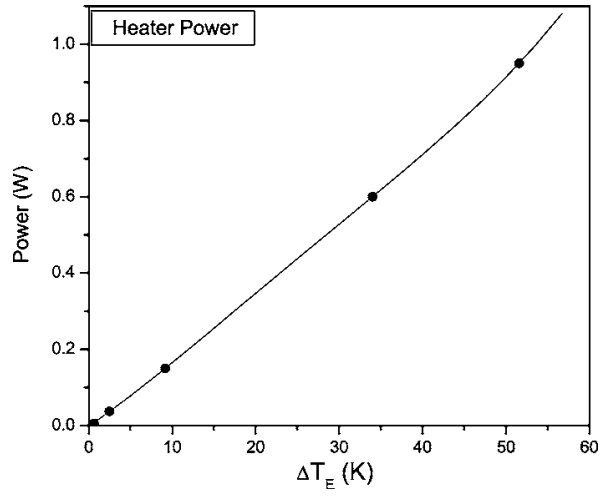

FIG. 3. Calibration curve of heater power vs $\Delta T_{E}$. The slope of the curve as $\Delta T_{E}$ tends to zero gives the value of $x$.

base plate. The heater block is supported by a thin walled quartz tube (5) of $10 \mathrm{~mm}$ diam and $100 \mathrm{~mm}$ length. This is provided with a spring loading (6) mounted on a stainlesssteel support frame (7). The quartz tube is used to minimize the conductive heat loss from the heater block to the support frame while the spring loading ensures proper contact between the sample and the holder assembly. The thermoelectric power is measured using two additional copper wires connected to the heater and base, respectively. The electrical insulation of the thermocouple wires prevents any electrical interference with these copper wires.

The central sample holder is placed inside a kanthal furnace (8) [15 mm inner diameter (i. d.) and $100 \mathrm{~mm}$ length] the height of which can be adjusted. Height adjustment of the furnace (9) can be done from the bottom in between a run without disturbing the sample. This has been done to minimize the temperature difference of the top and bottom thermocouple at the beginning of an experiment and also for easy loading and removal of the samples. A chromel-alumel thermocouple is embedded along with the heating element to control the furnace temperature through a multistep programmable temperature controller (Toho TTM-304).

A vacuum-tight glass chamber (10) $(80 \mathrm{~mm}$ diam and $400 \mathrm{~mm}$ height) encapsulates the whole arrangement to minimize convective heat exchange. The thermal contact between the heat source block and the sample is made or broken by a lifting mechanism (11). Using this, the central hot part of the sample holder is lifted by $0.5 \mathrm{~mm}$ under vacuum conditions.

\section{A. Sample preparation}

It is necessary to make samples of proper dimensions as the calculation of $\mathbf{K}$ depends on the sample dimensions. The samples used for measurement were either cylindrical or rectangular, and cut using a diamond cutter. In general, it was necessary to have an aspect ratio of $>10$ for successful experimentation. Extra care was taken to make the top and bottom surfaces parallel. Also, to ensure proper thermal contact with the heater and base, high-temperature silver paste was used as a contact material.

\section{B. Data collection}

The heater is energized by a Keithley 228 constant current source. The thermocouple temperatures are measured by

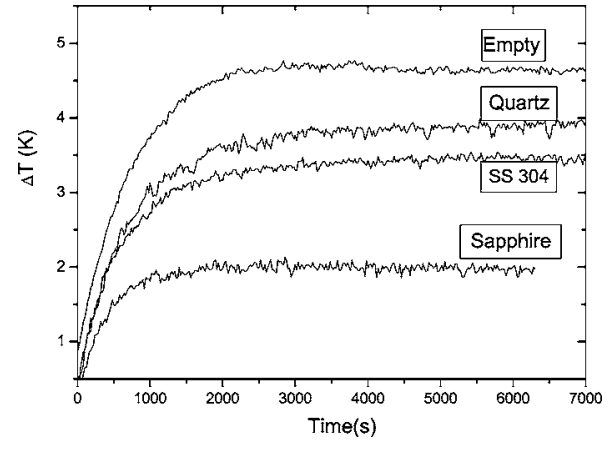

FIG. 4. Plot of $\Delta T$ vs time obtained for the different standard samples at room temperature.

a Keithley DMM 2700 scanner as a function of time. The current source and the multimeter outputs are interfaced to a computer using an IEEE-488 interface and the data collected in the hard disk using the commercial software XLINX 2700. The measurement consists of the following steps. Initially, the glass chamber is evacuated until a vacuum of $10^{-3}$ Torr is reached. The furnace is then heated to the desired temperature and allowed to stabilize. The furnace height is adjusted such that the top and bottom thermocouples read the same value. The constant current source is then put on and the change in temperature is measured as a function of time. Once the equilibrium $\Delta T_{S}$ value is reached, the heater is lifted slightly using the lifting mechanism and the change in temperature $\left(\Delta T_{E}\right)$ is again measured. It is important to repeat the empty run at each temperature as the heat loss keeps changing with temperature and also the furnace position.

\section{RESULTS AND DISCUSSION}

\section{A. Measurement of heater constant $(x)$}

To find the value of $x$, the $\Delta T_{E}$ of the heater is measured for different input power as shown in Fig. 3. $x$ is found from the slope of the curve as $\Delta T_{E}$ tends to zero. In the present case $x$ is $0.01491 \mathrm{~W} / \mathrm{K}$ at room temperature. For hightemperature measurements the value of $x$ has been modified depending on the change in specific heat of the heater block material (copper). A correction factor in $\Delta T_{E}$ has to be done for each temperature due to a change in the value of $x$. In the actual experiments $\Delta T$ was always maintained in the linear region of the calibration curve.

TABLE I. Thermal conductivity measurements of test materials at room temperature.

\begin{tabular}{lcccc}
\hline \hline \multirow{2}{*}{ Sample } & \multirow{2}{*}{$\begin{array}{c}\text { Height } \\
(\mathrm{cm})\end{array}$} & $\begin{array}{c}\text { Cross-sectional } \\
\text { area }\left(\mathrm{cm}^{2}\right)\end{array}$ & $\begin{array}{c}\text { Thermal conductivity } \\
( \pm 5 \%)\end{array}$ \\
\cline { 4 - 5 } & & & Observed & Reported \\
\hline Fused silica & 1.8 & 0.125 & 0.013 & 0.014 \\
SS 304 & 1.21 & 0.031 & 0.157 & 0.160 \\
Sapphire & 1.38 & 0.078 & 0.328 & 0.320 \\
\hline \hline
\end{tabular}




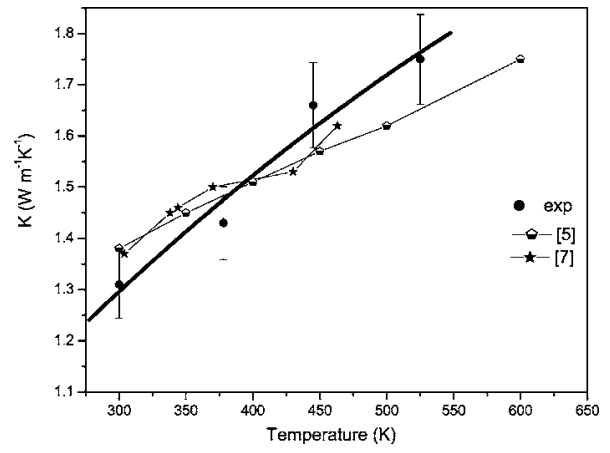

FIG. 5. Thermal conductivity of fused silica as a function of temperature. Plots [5], [7] are the experimental values taken from the literature.

\section{B. Room-temperature thermal conductivity measurements}

Test runs were carried out on standard samples and the data compared with literature data. Room-temperature thermal conductivity was measured for three different standard materials namely, sapphire, SS 304 and fused silica, which have wide variation in $\mathbf{K}$ values. The increase in temperature as a function of time for the different samples is shown in Fig. 4. The saturation $\Delta T$ could be achieved in sample dimensions and the observed and reported thermal conductivity values are given in Table I. It is seen from the data that a good match is observed with the data reported in the literature. ${ }^{5}$ Measurements on each sample were carried out three times and the deviation in the $\mathbf{K}$ value was within $\pm 5 \%$ of the absolute value.

\section{Measurement of $\mathrm{K}$ as a function of temperature}

Thermal conductivity was also measured as a function of temperature for fused silica as shown in Fig. 5. Fused silica has a very low thermal conductivity and the applicability of this technique for measurement of low $\mathbf{K}$ materials is demonstrated. The calculated data is compared with the data reported in the literature. ${ }^{5-8}$ Table II shows the scatter in the room-temperature data reported in the literature by various groups. This is because of the very low $\mathbf{K}$ value of fused silica. Our result lies well within the scatter region reported in the literature.

\section{SEEBECK COEFFICIENT MEASUREMENT}

The above-described apparatus can be used easily to measure the Seebeck coefficient at respective temperatures. For measuring the Seebeck coefficient $(\mathbf{S})$, the sample is placed between the source-sink assembly as shown in Fig 1(b). A constant input power is applied and the correspond-

TABLE II. Thermal conductivity data of fused silica at room temperature showing the acceptable scatter in data.

\begin{tabular}{cccccc}
\hline \hline Reference & 5 & 6 & 7 & 8 & Our data \\
\hline Temperature $(\mathrm{K})$ & 300 & 317 & 304 & 350 & 300 \\
$\mathrm{~K}\left(\mathrm{~W} \mathrm{~m}^{-1} \mathrm{~K}^{-1}\right)$ & 1.38 & 1.51 & 1.37 & 1.19 & 1.31 \\
\hline \hline
\end{tabular}

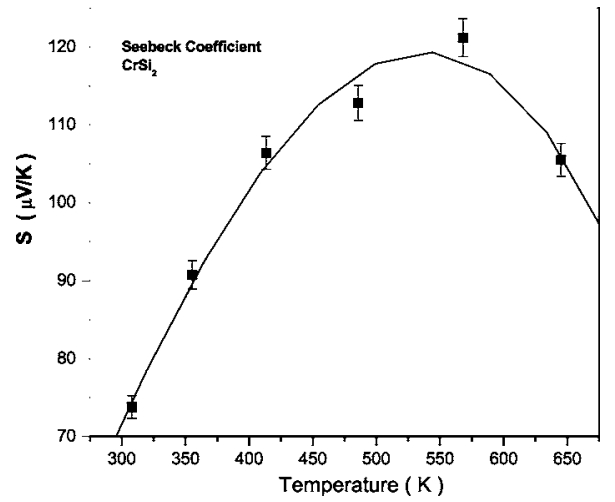

FIG. 6. Seebeck coefficient of $\mathrm{CrSi}_{2}$ as a function of temperature.

ing rise in temperature $\left(\Delta T_{S}\right)$ and voltage developed across the sample $(\Delta E)$ is measured. The Seebeck coefficient $\mathbf{S}$ can then be calculated using the equation

$$
S=\frac{\Delta E}{\Delta T_{S}},
$$

where $\Delta E$ is the voltage developed across the sample and $\Delta T_{S}$ is the corresponding rise in sample temperature. The absolute $\mathbf{S}$ values of the samples were obtained by applying the necessary correction due to the Seebeck coefficient of copper .

\section{MEASUREMENT OF S AND K VALUES FOR $\mathrm{CrSi}_{2}$}

$\mathrm{CrSi}_{2}$ has been used as a test thermoelectric material. The variation of $\mathbf{S}$ and $\mathbf{K}$ of the hot pressed sample with temperature is shown in Figs. 6 and 7, respectively. The sample was prepared by direct hot uniaxial pressing of elemental chromium and silicon powders. The thermoelectric data for $\mathrm{CrSi}_{2}$ as a function of temperature matches with the reported data by Nishida et al. ${ }^{9} \mathrm{Pure} \mathrm{CrSi}_{2}$ is $p$ type, as seen from the data. The $\mathbf{S}$ value increases initially with a maximum between 500 and $600 \mathrm{~K}$. The $\mathbf{K}$ was also measured as a function of temperature and is seen to decrease with increasing temperature. A detailed study on the thermoelectric properties of $\mathrm{CrSi}_{2}$ can be found elsewhere. ${ }^{10}$

In summary, we have designed and fabricated a setup to measure the thermal conductivity and thermoelectric power of small samples from room temperature to $700 \mathrm{~K}$. Equations have been developed based on the parallel conductance tech-

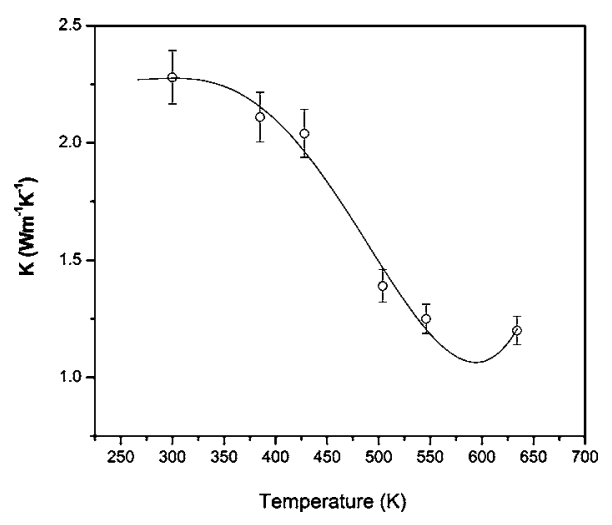

FIG. 7. Thermal conductivity of $\mathrm{CrSi}_{2}$ as a function of temperature. 
nique to measure $\mathbf{K}$. The $\mathbf{K}$ value measurements on test samples like sapphire, fused quartz, and stainless steel gave satisfactory results. The use of this apparatus for measuring both $\mathbf{K}$ and $\mathbf{S}$ values up to $700 \mathrm{~K}$ on $\mathrm{CrSi}_{2}$ has been demonstrated.

${ }^{1}$ M. J. Laubitz, Thermal Conductivity (Academic, London, 1969), Vol. 1, Chap. 3, p. 111.

${ }^{2}$ D. R. Flynn, Mechanical and thermal properties of ceramics, NBS Spec. Publication 303, p. 63 (1969).

${ }^{3}$ W. J. Parker, R. J. Jenkins, C. P. Butler, and G. L. Abbot, J. Appl. Phys.
32, 1679 (1961).

${ }^{4}$ B. M. Zawilski, R. T. Littleton, and T. M. Tritt, Rev. Sci. Instrum. 72, 1770 (2001).

${ }^{5}$ Thermophysical Properties of Matter, Vols. 1 \& 2 of Thermal Conductivity (IFI/Plenum, New York, 1970).

${ }^{6}$ J. H. Koenig, Rutgers Univ. N.J. Ceramic Research Station Progress Report, Vol. 2, 1 (1953).

${ }^{7}$ I. K. Kamilov, Sov. Phys. Solid State 4, 1693 (1963).

${ }^{8}$ K. L. Wray and T. J. Connolly, J. Appl. Phys. 30, 1702 (1959).

${ }^{9}$ I. Nishida and T. Sakata, J. Phys. Chem. Solids 39, 499 (1978).

${ }^{10}$ T Dasgupta and A. M. Umarji, communicated to Materials Research Bulletin (2005). 